\title{
New data on trematodes (Plathelminthes, Trematoda) of fishes in the Ross Sea (Antarctic)
}

\author{
S.G. Sokolov ${ }^{1}$, I.I. Gordeev ${ }^{2}$ \\ ${ }^{1}$ The Center of Parasitology IPEE RAS, Leninskyi Prospect. 33, Moscow 119071 Russia. e-mail: \\ sokolovsg@mail.ru \\ ${ }^{2}$ Russian Federal Research Institute of Fisheries and Oceanography (VNIRO), Verhnaya Kras- \\ noselskaya Str.17, Moscow107140 Russia.e-mail: gordeev@vniro.ru
}

ABSTRACT: Records of 13 trematode species, collected from bottom fishes from the western and northern parts of the Ross Sea, are given. Trematodes Otodistomum cestoides van Beneden, 1871, Lecithaster micropsi Zdzitowiecki, 1992, Neolepidapedon trematomi Prudhoe et Bray, 1973, Postlepidapedon opisthobifurcatum (Zdzitowiecki, 1990), Discoverytrema gibsoni Zdzitowiecki, 1990, D. markowskii Gibson, 1976, Macvicaria muraenolepidis Zdzitowiecki, 1990, Helicometra rakusai Zdzitowiecki, 1997 and Proctophantastes $\mathrm{sp}$. are recorded from the Ross Sea for the first time. Proctophantastes $\mathrm{sp}$. differs from other species of Proctophantastes Odhner, 1911 by the morphology of the ventral equatorial ridge of the ventral sucker.

How to cite this article: Sokolov S.G., Gordeev I.I. 2013. New data on trematodes (Plathelminthes, Trematoda) of fishes in the Ross Sea (Antarctic) // Invert. Zool. Vol.10. No.2. P.255-267.

KEY WORDS: the Ross Sea, Antarctic, trematodes, Nototheniidae, Rajidae, Channichthyidae, Macrouridae, Muraenolepididae, Zoogonidae, Proctophantastes.

\section{Новые данные о трематодах (Plathelminthes, Trematoda) рыб моря Росса (Антарктика)}

\section{С.Г. Соколов ${ }^{1}$, И.И. Гордеев²}

\footnotetext{
${ }^{1}$ Центр паразитологии ИПЭЭ РАН, Ленинский проспект 33, Москва 119071, Россия. е-таil: sokolovsg@mail.ru

${ }^{2}$ Всероссийский институт рыбного хозяйства и океанографии (ВНИРО), ул. Верхняя Красносельская 17, Москва 107140, Россия.e-mail: gordeev@vniro.ru
}

РЕЗЮМЕ: Дан обзор 13 видов трематод, собранных от донных рыб западной и северной частей моря Росса. Трематоды Otodistomum cestoides (van Beneden, 1871), Lecithaster micropsi Zdzitowiecki, 1992, Neolepidapedon trematomi Prudhoe et Bray, 1973, Postlepidapedon opisthobifurcatum (Zdzitowiecki, 1990), Discoverytrema gibsoni Zdzitowiecki, 1990,D. markowskii Gibson, 1976, Macvicaria muraenolepidis Zdzitowiecki, 1990, Helicometra rakusai Zdzitowiecki, 1997 и Proctophantastes sp. впервые указываются для моря Росcа. Proctophantastes sp. отличается от других видов соответствующего рода морфологией вентрального экваториального гребня на брюшной присоске. 
Как цитировать эту статью: Sokolov S.G., Gordeev I.I. 2013. New data on trematodes (Plathelminthes, Trematoda) of fishes in the Ross Sea (Antarctic) // Invert. Zool. Vol.10. No.2. P.255-267.

КЛЮЧЕВЫЕ СЛОВА: море Росса, Антарктика, трематоды, Nototheniidae, Rajidae, Channichthyidae, Macrouridae, Muraenolepididae, Zoogonidae, Proctophantastes.

\section{Introduction}

The first information on fish parasites from the Ross Sea were published by Leiper, Atkinson $(1914,1915)$ on the basis of material collected during the British Antarctic "Terra Nova" Expedition of 1910-1913. By the end of 2012 data on 17 species and forms of trematodes undetermined to the species level (taking into account generally recognized synonymy) were collected from 16 fish species in the Ross Sea (Byrd, 1963; Holloway, Bier, 1968; Holloway, Spence, 1980; Orecchia, Paggi, 1990; Moser, Cowen, 1991; Zdzitowiecki et al., 1999; Brickle et al., 2005; Laskowski et al., 2005). These investigations were conducted in Terra Nova Bay and McMurdo Sound near land stations. However, the ichthyofauna of the Ross Sea comprises more than 80 species of fishes (Andriyashev, 1986; Eastman, Hubold, 1999; Donnelly et al., 2004), suggesting a rich fauna of trematodes from fishes in this area.

The aim of the present work was to record and describe trematodes from bottom fishes caught during longline fishing for the Antarctic toothfish Dissostichus mawsoni Norman, 1937 in the western and northern parts of the Ross Sea.

\section{Materials and methods}

Fishing was carried out by both the Russian longliner "Yantar-31" using autoline fishing gear from December 2011 to February 2012 and the Korean longliner "Insung No.7" using trotline fishing gear from December 2010 to February 2011. Eighty six specimens of Dissostichus mawsoni (fam. Nototheniidae) (total length [TL] $75-178 \mathrm{~cm})$ were examined, as were six other species caught as by-catches. These were:
Bathyraja meridionalis Stehmann, 1987 (fam. Rajidae, seven specimens, TL $=53-131 \mathrm{~cm}$ ), Chionobathyscus dewitti Andriashev et Neyelov, 1978 (fam. Channichthyidae, 16 specimens, TL =23-40 cm), Macrourus whitsoni (Regan, 1913) (fam. Macrouridae, 76 specimens, $\mathrm{TL}=40-115$ $\mathrm{cm})$, Trematomus hansoni Boulenger, 1902 (fam. Nototheniidae, 49 specimens, $\mathrm{TL}=15-29$ $\mathrm{cm}$ ), Pogonophryne sp. (fam. Artedidraconidae, 15 specimens, TL $=24-31 \mathrm{~cm}$ ) and Muraenolepis marmorata Günther, 1880 (fam. Muraenolepididae, 56 specimens, $\mathrm{TL}=27-51 \mathrm{~cm}$ ). Because of the fact that the international community is concerned about the fate of skates, $B$. meridionalis individuals caught alive were picked up for examination only with if they had injuries incompatible with life, according to the CCAMLR (Commission for the Conservation of Marine Living Resources) Conservation Measures (CCAMLR, 2012). Fish identification was carried out using Fisher, Hureau (1985) and the "Scientific Observer Manual" (CCAMLR, 2011).

All fishes were examined for parasitic infestation using the standard method of Bykhovskaya-Pavlovskaya(1985). Alive worms were fixated under slight coverslip pressure in $70 \%$ ethanol. Samples were stained with acetocarmine and mounted in Canada balsam.

Data on the species of trematodes are given with the host and site of infection. Values for prevalence and intensity are given in the parentheses following the locality data as: (number of fishes infected/number examined, intensity of infection) (Bush et al., 1997). The classification of trematodes follows the "Keys to the Trematoda" (Gibson et al., 2002; Jones et al., 2005; Bray et al., 2008), with additions from Bray, Cribb (2012). Families and species are listed in alphabetical order. For the species that are found 
for the first time in the Ross Sea we provide original figures and body and organs metrical data. This confirms adequacy of species identification. Drawings were made with the aid of a drawing tube. Voucher specimens have been deposited in the Helminthological Museum of the Center of Parasitology (HMCP), IPEE RAS, Moscow.

\section{Results}

We have found 13 species of trematodes.

Family AZYGIIDAE Lühe, 1909

Subfamily AZYGIINAE Lühe, 1909

Genus Otodistomum Stafford, 1904

Otodistomum cestoides (van Beneden, 1871) Fig. 1A; Table 1.

Numbers of voucher specimens in HMCP: 3987/Tr.

Host: Bathyraja meridionalis.

Site of infection: spiral valve.

Locality: Ross Sea, $77^{\circ} \mathrm{S}, 178^{\circ} \mathrm{W}(7 / 7,1-57)$.

Previous records from the Ross Sea: none.

Other localities in Antarctic waters: near the South Shetland Islands (Zdzitowiecki, 1991), the Weddell Sea (Zdzitowiecki, 1997).

\section{Family DEROGENIDAE Nicoll, 1910}

Subfamily GONOCERCINAE Skrjanin et Guschanskaja, 1955

Genus Gonocerca Manter, 1925

Gonocerca phycidis Manter, 1925

Numbers of voucher specimens in HMCP: 3941/Tr.

Host: Muraenolepis marmorata.

Site of infection: intestine.

Locality: Ross Sea $75^{\circ} \mathrm{S} 174^{\circ} \mathrm{W}(1 / 9,1)$.

Previous records from the Ross Sea: Byrd (1963), Orecchia, Paggi, (1990), Zdzitowiecki et al. (1999).

\section{Family LECITHASTERIDAE Odhner, 1905}

Subfamily Lecithasterinae Lühe, 1901

Genus Lecithaster Lühe, 1901

Lecithaster micropsi Zdzitowiecki, 1992

Fig. 1B; Table 1.

Numbers of voucher specimens in HMCP: 3997/Tr - 3941/Tr.

Host: Muraenolepis marmorata.

Site of infection: intestine.

Locality: Ross Sea $75^{\circ} \mathrm{S} 174^{\circ} \mathrm{W}(1 / 9,28)$.

Previous records from the Ross Sea: none.

Other localities in Antarctic waters: none; is known from the Atlantic sector of sub-Antarctic - near South Georgia Island (Zdzitowiecki, 1992; Zdzitowiecki et al., 1997).

\section{Family LEPIDAPEDIDAE Yamaguti, 1958}

Genus Lepidapedon Stafford, 1904

Lepidapedon garrardi Leiper et Atkinson, 1914

Numbers of voucher specimens in HMCP: 3985/Tr, 3986/Tr.

Host: Trematomus hansoni.

Site of infection: intestine.

Locality: Ross Sea $77^{\circ} \mathrm{S} 178^{\circ} \mathrm{W}(1 / 49,5)$.

Previous records from the Ross Sea: Leiper, Atkinson (1914, 1915); Byrd (1963); Holloway, Spence (1980); Orecchia, Paggi (1990); Moser, Cowen (1991); Zdzitowiecki et al. (1999).

Genus Neolepidapedon Manter, 1954 1973

Neolepidapedon trematomi Prudhoe et Bray, Fig. 1C; Table 1.

Numbers of voucher specimens in HMCP: 3988/Tr.

Host: Dissostichus mawsoni, Chionobathyscus dewitti. 
Table 1. Measurements of trematodes from fishes of the Ross Sea: Azygiidae, Lecithasteridae and Lepidapedidae.

Таблица 1. Измерения трематод из рыб моря Росса: Azygiidae, Lecithasteridae и Lepidapedidae.

\begin{tabular}{|c|c|c|c|c|c|}
\hline \multirow[b]{2}{*}{ Characters } & \multicolumn{5}{|c|}{ Species } \\
\hline & $\begin{array}{l}\text { Otodistomum } \\
\text { cestoides, } \mathrm{n}=1\end{array}$ & $\begin{array}{l}\text { Lecithaster } \\
\text { micropsi, } \mathrm{n}=5\end{array}$ & $\begin{array}{l}\text { Neolepidapedon } \\
\text { trematomi, } \mathrm{n}=2\end{array}$ & $\begin{array}{l}\text { Paralepidapedon } \\
\text { cf. dubium, } \mathrm{n}=6\end{array}$ & $\begin{array}{l}\text { Postlepidapedon } \\
\text { opisthobifurcatum, } \\
\mathrm{n}=4\end{array}$ \\
\hline Host & $\begin{array}{l}\text { Bathyraja } \\
\text { meridionalis }\end{array}$ & $\begin{array}{l}\text { Muraenolepis } \\
\text { marmorata }\end{array}$ & $\begin{array}{l}\text { Dissostichus } \\
\text { mawsoni }\end{array}$ & M. marmorata & $\begin{array}{l}\text { Macrourus } \\
\text { whitsoni and } M . \\
\text { marmorata }\end{array}$ \\
\hline $\begin{array}{l}\text { Body: Length (L, } \\
\text { mm) }\end{array}$ & 38.78 & $1.20-1.69$ & $2.20-2.39$ & $1.76-2.61$ & $1.94-2.49$ \\
\hline $\begin{array}{l}\text { Body: Width (W, } \\
\text { mm) }\end{array}$ & 3.45 & $0.52-0.79$ & $0.79-0.90$ & $0.47-0.57$ & $0.52-0.71$ \\
\hline $\begin{array}{l}\text { Forebody: } \% \text { of } \\
\text { body length }\end{array}$ & 12.0 & $18.0-25.5$ & $32.2-39.2$ & $25.3-28.7$ & $25.7-31.6$ \\
\hline $\begin{array}{l}\text { Posttesticular space: } \\
\% \text { of body length }\end{array}$ & 46.6 & $34.5-36.4$ & $20.8-23.8$ & $17.2-20.8$ & $18.4-22.8$ \\
\hline Oral sucker: L & 1.114 & $0.179-0.221$ & $0.200-0.210$ & $0.135-0.175$ & $0.210-0.252$ \\
\hline Oral sucker: W & 1.190 & $0.168-0.231$ & $0.210-0.221$ & $0.153-0.188$ & $0.200-0.257$ \\
\hline Prepharynx: L & - & - & $0.011-0.063$ & $0.074-0.110$ & $0.074-0.195$ \\
\hline Pharynx: L & 0.576 & $0.147-0.153$ & 0.116 & $0.135-0.161$ & $0.126-0.180$ \\
\hline Pharynx: W & 0.422 & $0.117-0.141$ & $0.084-0.085$ & $0.080-0.121$ & $0.095-0.137$ \\
\hline Oesophagus: L & 0.422 & - & $0.210-0.221$ & $0.074-0.190$ & $0.300-0.347$ \\
\hline Ventral sucker: L & 1.555 & $0.294-0.420$ & 0.242 & $0.178-0.202$ & $0.200-0.263$ \\
\hline Ventral sucker: W & 1.670 & $0.336-0.420$ & $0.247-0.252$ & $0.190-0.215$ & $0.200-0.289$ \\
\hline $\begin{array}{l}\text { Anterior or left } \\
\text { testis: L }\end{array}$ & 1.286 & $0.153-0.215$ & $0.210-0.221$ & $0.153-0.235$ & $0.200-0.301$ \\
\hline $\begin{array}{l}\text { Anterior or left } \\
\text { testis: W }\end{array}$ & 1.114 & $0.141-0.276$ & $0.221-0.252$ & $0.153-0.215$ & $0.231-0.326$ \\
\hline $\begin{array}{l}\text { Posterior or right } \\
\text { testis: L }\end{array}$ & 1.440 & $0.172-0.245$ & $0.252-0.273$ & $0.184-0.242$ & $0.242-0.368$ \\
\hline $\begin{array}{l}\text { Posterior or right } \\
\text { testis: W }\end{array}$ & 1.056 & $0.153-0.251$ & $0.257-0.315$ & $0.172-0.215$ & $0.210-0.368$ \\
\hline Cirrus sac: L & - & - & - & $0.137-0.168$ & $0.153-0.186$ \\
\hline Cirrus sac: W & - & - & 0.116 & $0.079-0.088$ & $0.044-0.061$ \\
\hline Ovary: L & 1.152 & $0.245-0.307$ & $0.179-0.242$ & $0.172-0.258$ & $0.147-0.189$ \\
\hline Ovary: W & 1.094 & $0.264-0.405$ & $0.168-0.179$ & $0.153-0.221$ & $0.137-0.179$ \\
\hline Vitellarium: L & - & $0.258-0.393$ & - & - & - \\
\hline Vitellarium: W & - & $0.288-0.466$ & - & - & - \\
\hline Eggs: L, $\mu \mathrm{m}$ & 72 & 31 & $82-85^{*}$ & $64-73 *$ & 88-93* \\
\hline Eggs: $\mathrm{W}, \mu \mathrm{m}$ & 49 & 18 & - & - & - \\
\hline
\end{tabular}

* eggs are deformed in balsam

Site of infection: intestine.

Locality: Ross Sea $75^{\circ} \mathrm{S} 174^{\circ} \mathrm{W}(D$. mawso$n i-1 / 25,3$ and $C$. dewitti $-1 / 16,2)$ and $75^{\circ} \mathrm{S}$ $175^{\circ} \mathrm{W}($ D. mawsoni - $1 / 22,24)$.
Previous records from the Ross Sea: none. Other localities in Antarctic waters: near the South Shetland Islands (Zdzitowiecki, 1987, 1988, 1991, 1999; Zdzitowiecki et al., 1997; 


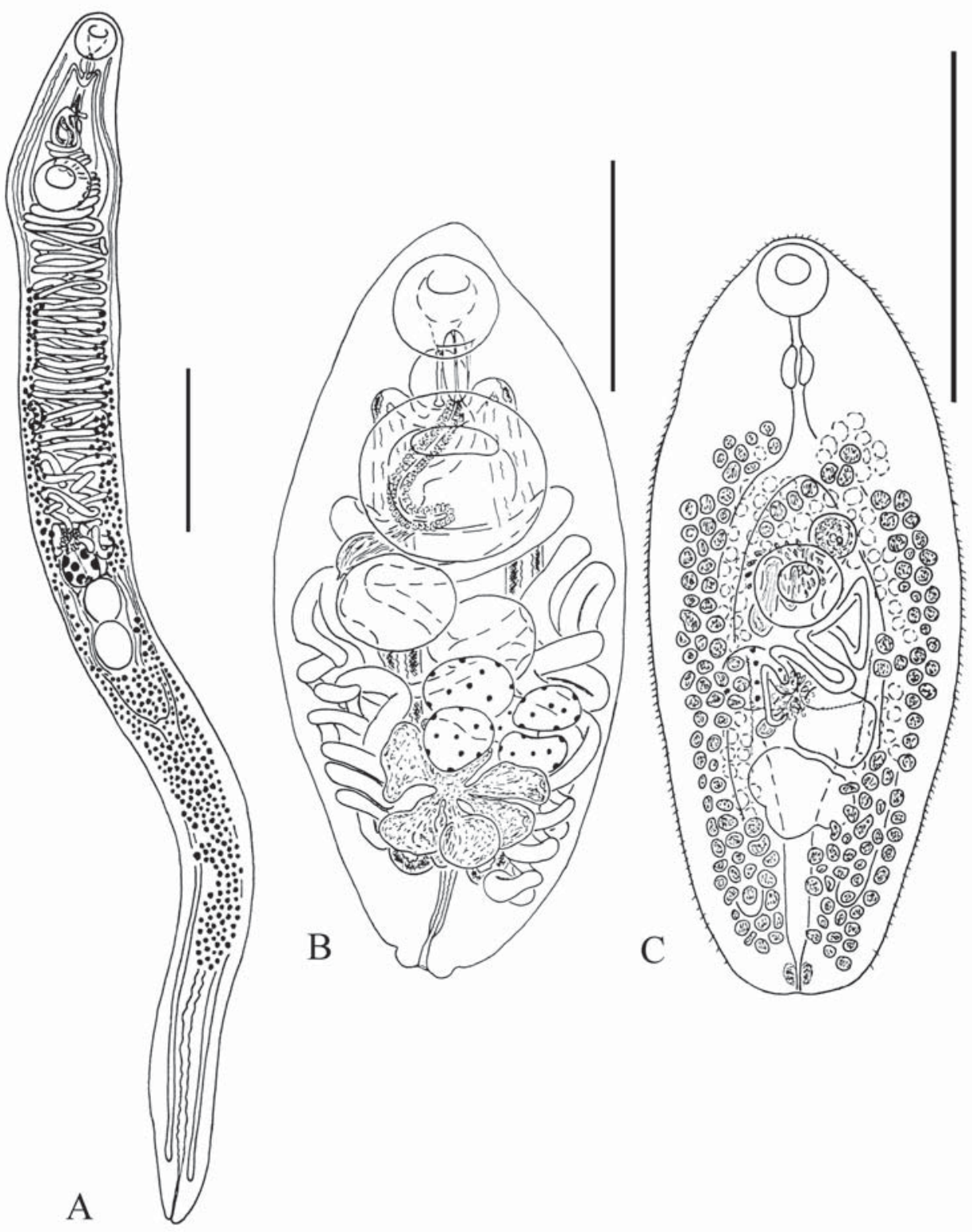

Fig. 1. Trematodes of fam. Azygiidae, Lecithasteridae and Lepidapedidae.

A - Otodistomum cestoides, general view; B - Lecithaster micropsi, general view; C - Neolepidapedon trematomi, general view. Scale bars: A $-5 \mathrm{~mm}$; B $-0.5 \mathrm{~mm}$; C $-1 \mathrm{~mm}$.

Рис. 1. Трематоды семейств Azygiidae, Lecithasteridae и Lepidapedidae.

A — Otodistomum cestoides, общий вид; В — Lecithaster micropsi, общий вид; C — Neolepidapedon trematomi, общий вид. Масштаб: А -5 мм; В $-0,5$ мм; С -1 мм.

Zdzitowiecki, Laskowski, 2004), off Adelie Land (Zdzitowiecki, 2001), the Weddell Sea
(Zdzitowiecki, Cielecka, 1997b); the Indian Ocean sector (Prudhoe, Bray, 1973). 
Genus Paralepidapedon Shimazu et Shimura, 1984

Paralepidapedon cf. dubium Prudhoe et Bray, 1973

Fig. 2A; Table 1.

Numbers of voucher specimens in HMCP: 3995/Tr, 3996/Tr.

Host: Muraenolepis marmorata.

Site of infection: intestine.

Locality: Ross Sea $72^{\circ} \mathrm{S} 175^{\circ} \mathrm{W}(1 / 42,5)$ and $75^{\circ} \mathrm{S} 174^{\circ} \mathrm{W}(1 / 9,10)$.

Previous records from the Ross Sea: none.

REMARK. Morphological characters of Paralepidapedon cf. dubium correspond to the description of Paralepidapedon dubium Prudhoe et Bray, 1973 except the structure of an external seminal vesicle. The external seminal vesicle in studied specimens is surrounded by prostatical cells which are enclosed in membranous sac. The external seminal vesicle with no associated prostatical cells in P. dubium. Species $P$. dubium was described from $\mathrm{Mac}$ rourus whitsoni from the Collaboration Sea (the Indian Ocean sector of Antarctic) (Prudhoe, Bray, 1973).

Genus Postlepidapedon Zdzitowiecki, 1993

Postlepidapedon opisthobifurcatum (Zdzitowiecki, 1990)

Fig. 2B; Table 1.

Numbers of voucher specimens in HMCP: 3996/Tr.

Host: Muraenolepis marmorata, Macrourus whitsoni.

Site of infection: intestine.

Localities: Ross Sea $72^{\circ} \mathrm{S} 175^{\circ} \mathrm{W}$ (M. marmorata $-2 / 42,1)$ and $75^{\circ} \mathrm{S} 175^{\circ} \mathrm{W}$ (M. whitso$n i-1 / 38,4)$.

Previous records from the Ross Sea: none.

Other localities in Antarctic waters: the Weddell Sea (Zdzitowiecki, Cielecka, 1997a; Walter et al., 2002).
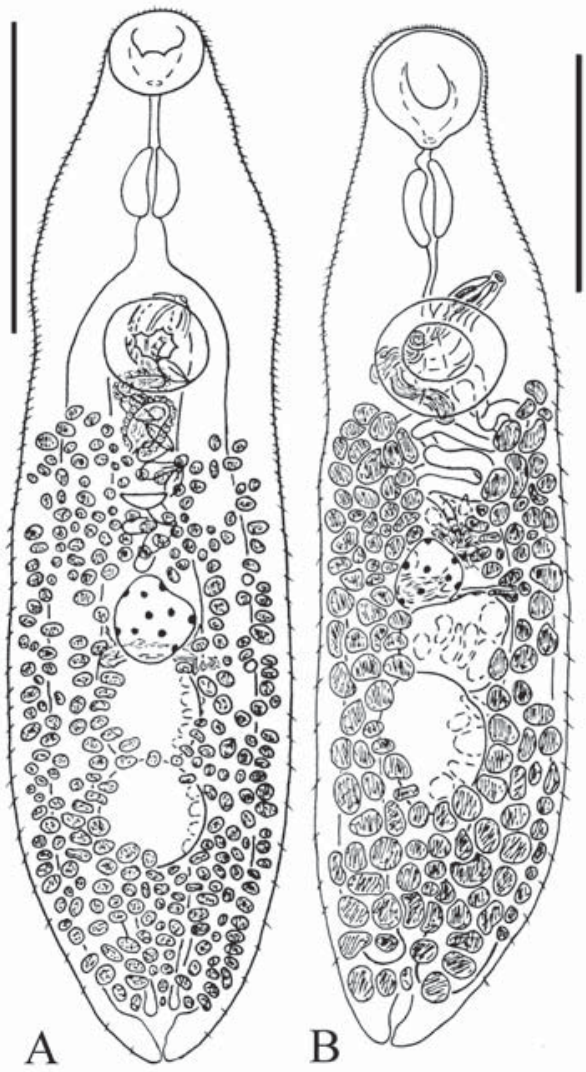

Fig. 2. Trematodes of fam. Lepidapedidae.

A - Paralepidapedon cf. dubium, general view; B Postlepidapedon opisthobifurcatum, general view. Scale bars: A, B $-0.5 \mathrm{~mm}$.

Рис. 2. Трематоды семейства Lepidapedidae.

A - Paralepidapedon cf. dubium, общий вид; В Postlepidapedon opisthobifurcatum, общий вид. Масштаб: А, В - 0,5 мм.

\section{Family OPECOELIDAE Ozaki, 1925}

Subfamily OPECOELINAE Ozaki, 1925

Genus Discoverytrema Gibson, 1976

Discoverytrema gibsoniZdzitowiecki, 1990 Fig.3 A, B; Table 2.

Numbers of voucher specimens in HMCP: 3942/Tr - 3944/Tr.

Host: Muraenolepis marmorata.

Site of infection: intestine. 

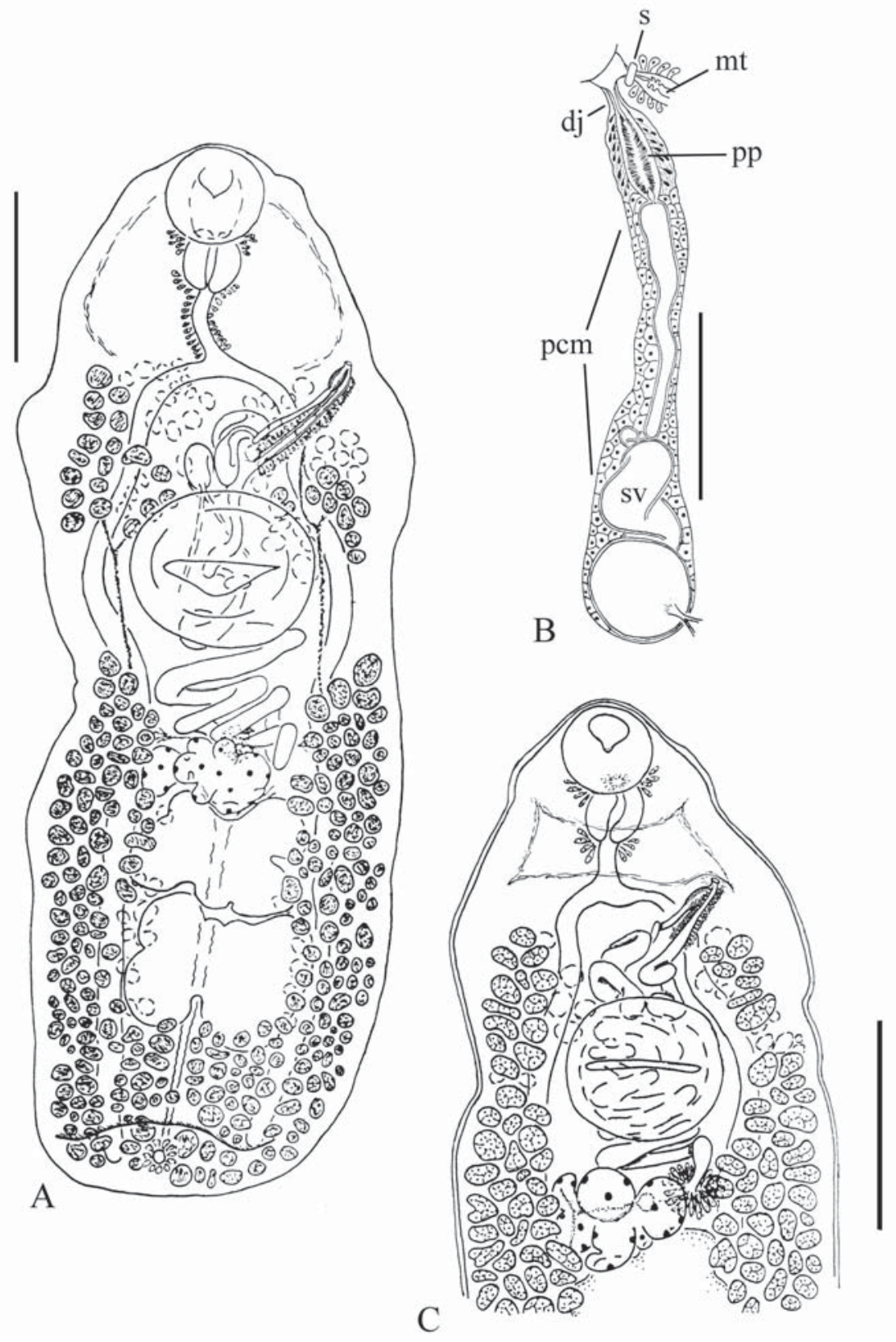

Fig. 3. Trematodes of gen. Discoverytrema.

A - D. gibsoni, general view; B - terminal genital apparatus of subadult specimens D. gibsoni; $\mathrm{C}-D$. markowskii, general view. Scale bars: A $-0.5 \mathrm{~mm} ; \mathrm{B}-0.15 \mathrm{~mm}$; $-0.6 \mathrm{~mm}$. Abbreviations: $\mathrm{s}-$ sphincter, $\mathrm{mt}$ - metraterm, pp - pars prostatica, dj - ejaculatory duct, sv - seminal vesicle, $\mathrm{pcm}$ - prostatical cells which are enclosed in membranous sac.

Fig 3. Трематоды рода Discoverytrema.

A - D. gibsoni, общий вид; В - терминальная часть полового аппарата молодой мариты $D$. gibsoni; $\mathrm{C}-D$. markowskii, общий вид. Масштаб: А - 0,5 мм; В - 0,15 мм; C - 0,6 мм. Обозначения: $\mathrm{s}-$ сфинктер, $\mathrm{mt}-$ метратерм, pp - простатическая часть, dj - семяизвергательный канал, sv - семянной пузырек, pcm простатические клетки, заключенные в пленчатый мешок. 
Table 2. Measurements of trematodes from fishes of the Ross Sea: Opecoelidae and Zoogonidae. Таблица 2. Измерения трематод из рыб моря Росса: Opecoelidae и Zoogonidae.

\begin{tabular}{|l|l|l|l|l|l|}
\hline \multirow{2}{*}{ Characters } & \multicolumn{5}{|c|}{ Species } \\
\cline { 2 - 6 } & $\begin{array}{l}\text { Discoverytrema } \\
\text { gibsoni, } \mathrm{n}=6\end{array}$ & $\begin{array}{l}\text { D. markowskii, } \\
\mathrm{n}=3\end{array}$ & $\begin{array}{l}\text { Helicometra } \\
\text { rakusai, } \mathrm{n}=1\end{array}$ & $\begin{array}{l}\text { Macvicaria } \\
\text { muraenolepidis, } \\
\mathrm{n}=3\end{array}$ & $\begin{array}{l}\text { Proctophantastes } \\
\text { sp., n=3 }\end{array}$ \\
\hline Host & $\begin{array}{l}\text { Muraenolepis } \\
\text { marmorata }\end{array}$ & M. marmorata & $\begin{array}{l}\text { Dissostichus } \\
\text { mawsoni }\end{array}$ & M. marmorata & M. marmorata \\
\hline $\begin{array}{l}\text { Body: Length (L, } \\
\text { mm) }\end{array}$ & $2.11-3.39$ & $2.51-2.76$ & 1.79 & $1.12-1.23$ & $1.94-2.35$ \\
\hline $\begin{array}{l}\text { Body: Width (W, } \\
\text { mm) }\end{array}$ & $0.77-1.37$ & $1.03-1.12$ & 0.46 & $0.35-0.39$ & $0.93-1.21$ \\
\hline $\begin{array}{l}\text { Forebody: \% of } \\
\text { body length }\end{array}$ & $29.6-37.8$ & $30.7-35.6$ & 26.8 & $29.1-34.6$ & $43.4-46.5$ \\
\hline $\begin{array}{l}\text { Posttesticular } \\
\text { space: \% of body } \\
\text { length }\end{array}$ & $15.3-20.6$ & $12.3-21.4$ & 24.4 & $8.4-9.4$ & $22.9-23.1$ \\
\hline Oral sucker: L & $0.200-0.273$ & $0.226-0.252$ & 0.186 & $0.121-0.137$ & $0.294-0.378$ \\
\hline Oral sucker: W & $0.200-0.315$ & $0.231-0.279$ & 0.236 & $0.126-0.137$ & $0.357-0.389$ \\
\hline Prepharynx: L & $\sim 0.040$ & - & - & $\sim 0.006$ & - \\
\hline Pharynx: L & $0.137-0.184$ & $0.158-0.179$ & 0.107 & $0.084-0.090$ & $0.129-0.144$ \\
\hline Pharynx: W & $0.131-0.189$ & $0.147-0.168$ & 0.122 & $0.060-0.072$ & $0.074-0.080$ \\
\hline Oesophagus: L & $0.126-0.263$ & $0.116-0.158$ & 0.063 & 0.060 & $0.129-0.147$ \\
\hline Ventral sucker: L & $0.336-0.452$ & $0.305-0.389$ & 0.179 & $0.221-0.273$ & $0.279-0.393 \mathrm{x}$ \\
\hline Ventral sucker: W & $0.357-0.531$ & $0.252-0.452$ & 0.179 & $0.326-0.0331$ & $0.788-0.967$ \\
\hline $\begin{array}{l}\text { Anterior or left } \\
\text { testis: L }\end{array}$ & $0.184-0.399$ & $0.258-0.337$ & 0.193 & $0.147-0.189$ & $0.245-0.374 \mathrm{x}$ \\
\hline $\begin{array}{l}\text { Anterior or left } \\
\text { testis: W }\end{array}$ & $0.399-0.570$ & $0.429-0.540$ & 0.157 & $0.179-0.221$ & $0.258-0.368$ \\
\hline $\begin{array}{l}\text { Posterior or right } \\
\text { testis: L }\end{array}$ & $0.313-0.460$ & $0.288-0.429$ & 0.215 & 0.200 & $0.368-0.374$ \\
\hline $\begin{array}{l}\text { Posterior or right } \\
\text { testis: W }\end{array}$ & $0.356-0.570$ & $0.466-0.527$ & 0.157 & $0.189-0.200$ & $0.245-0.307$ \\
\hline Cirrus sac: L & - & - & 0.350 & $0.279-0.294$ & $0.494-0.541$ \\
\hline Cirrus sac: W & - & - & 0.107 & $0.067-0.072$ & $0.189-0.231$ \\
\hline Ovary: L & $0.184-0.270$ & $0.305-0.389$ & 0.164 & $0.116-0.147$ & $0.221-0.319$ \\
\hline Ovary: W & $0.331-0.410$ & $0.252-0.452$ & 0.150 & $0.158-0.200$ & $0.215-0.273$ \\
\hline Eggs: L, $\mu \mathrm{m}$ & $58-64 *$ & $54-62 *$ & $\begin{array}{l}68-70 * \\
\text { (without a } \\
\text { filament) }\end{array}$ & $41-44 *$ & $37-40$ \\
\hline
\end{tabular}

* eggs are deformed in balsam

Locality: Ross Sea $72^{\circ} \mathrm{S} 175^{\circ} \mathrm{W}(2 / 42,4-5)$ and $75^{\circ} \mathrm{S} 174^{\circ} \mathrm{W}(1 / 9,18)$.

Previous records from the Ross Sea: none.

Other localities in Antarctic waters: near the South Shetland Islands ( Palm et al., 2007).
Discoverytrema markowskii Gibson, 1976 Fig. 3C; Table 2.

Numbers of voucher specimens in HMCP: 3943/Tr, 3944/Tr.

Host: Muraenolepis marmorata. 


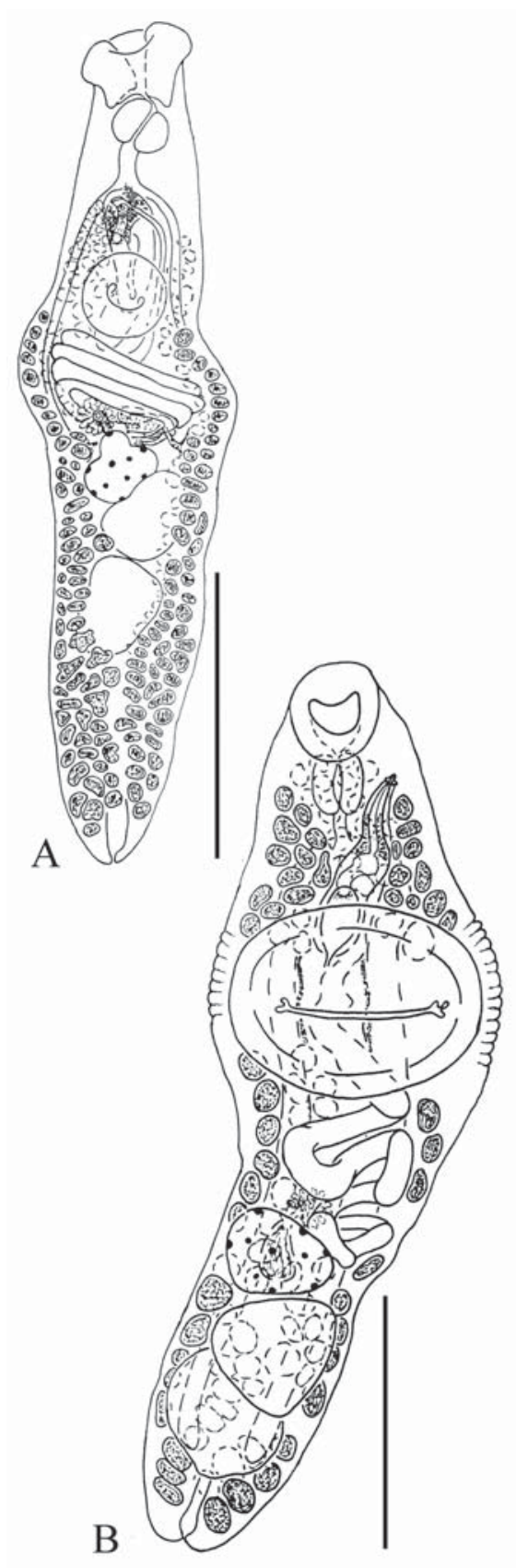

Fig. 4. Trematodes of fam. Opecoelidae.

A - Helicometra rakusai, general view; B - Macvicaria muraenolepidis, general view. Scale bars: A $-0.6 \mathrm{~mm}$; B $-0.5 \mathrm{~mm}$

Fig. 4. Трематоды семейства Opecoelidae. A - Helicometra rakusai, общий вид; В - Macvicaria muraenolepidis, общий вид. Масштаб: А-0,6 мм; В0,5 мм
Site of infection: intestine.

Locality: Ross Sea $72^{\circ} \mathrm{S} 175^{\circ} \mathrm{W}(1 / 42,4)$.

Previous records from the Ross Sea: none.

Other localities in Antarctic waters: near the South Shetland Islands (Gaevskaya, Rodjuk, 1997; Palm et al., 2007).

\section{Family OPECOELIDAE Ozaki, 1925}

Subfamily PLAGIOPORINAE Manter, 1947

Genus Helicometra Odhner, 1902

Helicometra antarcticae Holloway et Bier, 1968

Numbers of voucher specimens in HMCP: 3989/Tr - 3993/Tr.

Host: Dissostichus mawsoni.

Site of infection: intestine.

Localities: Ross Sea $75^{\circ} \mathrm{S} 174^{\circ} \mathrm{W}(3 / 25,7-$ $1182), 75^{\circ} \mathrm{S} 175^{\circ} \mathrm{W}(1 / 22,8)$ and $76^{\circ} \mathrm{S} 170^{\circ} \mathrm{W}$ $(1 / 25,3)$.

Previous records from the Ross Sea: Holloway, Bier, (1968); Holloway, Spence (1980); Brickle et al., (2005).

Helicometra rakusai Zdzitowiecki, 1997

Fig. 4A; Table 2.

Numbers of voucher specimens in HMCP: 3945/Tr.

Host: Dissostichus mawsoni.

Site of infection: intestine.

Locality: Ross Sea $75^{\circ} \mathrm{S} 174^{\circ} \mathrm{W}(1 / 25,1)$.

Previous records from the Ross Sea: none.

Other localities in Antarctic waters: the Weddell Sea (Zdzitowiecki, 1997).

Genus Macvicaria Gibson et Bray, 1982

Macvicaria muraenolepidis Zdzitowiecki, 1990

Fig. 4B; Table 2.

Numbers of voucher specimens in HMCP: 3995/Tr.

Host: Muraenolepis marmorata. 
Site of infection: intestine.

Locality: Ross Sea $72^{\circ} \mathrm{S} 175^{\circ} \mathrm{W}(1 / 42,9)$.

Previous records from the Ross Sea: none.

Other localities in Antarctic waters: none; is known from the Atlantic sector of sub-Antarctic — near South Georgia Island (Zdzitowiecki, 1990).

\section{Family ZOOGONIDAE Odhner, 1902}

\author{
Subfamily LEPIDOPHYLLINAE Stossich, \\ 1903
}

Genus Proctophantastes Odhner, 1911

\section{Proctophantastes sp.}

Fig. 5; Table 2.

Numbers of voucher specimens in HMCP: 3994/Tr.

Host: Muraenolepis marmorata

Site of infection: intestine.

Locality: Ross Sea $75^{\circ} \mathrm{S} 174^{\circ} \mathrm{W}(1 / 9,3)$.

Previous records from the Ross Sea: none.

Other localities in Antarctic waters: none.

REMARK. Body of the examined trematodes pyriform, with numerous tegumental spines and subtegumentary glands. Oral sucker globular or subglobular, subterminal. Large ventral sucker equatorial, transversely elongate, with horizontal median dorsal cleft and ventral ridge; latter composed of subglobular or oblong muscular protrusions closely adjacent to one another. Prepharynx not visible; pharynx present; oesophagus short. Pharynx and oesophagus surrounded by glandular cells. Intestinal bifurcation slightly posterior to mid-forebody. Caeca end blindly near testes in hindbody. Testes two in hindbody. suboval, symmetrical. Cirrus sac claviform, transversal; proximal end of cirrus sac at level of junction of first and second thirds of ventral sucker length, or just posterior to this. Cirrus sac together with metraterm opens into common genital atrium, which is surrounded by lobed periatrial gland together with metraterm opens into common genital atrium, which is surrounded by lobed periatrial gland. Genital pore submarginal on left side of body, slightly

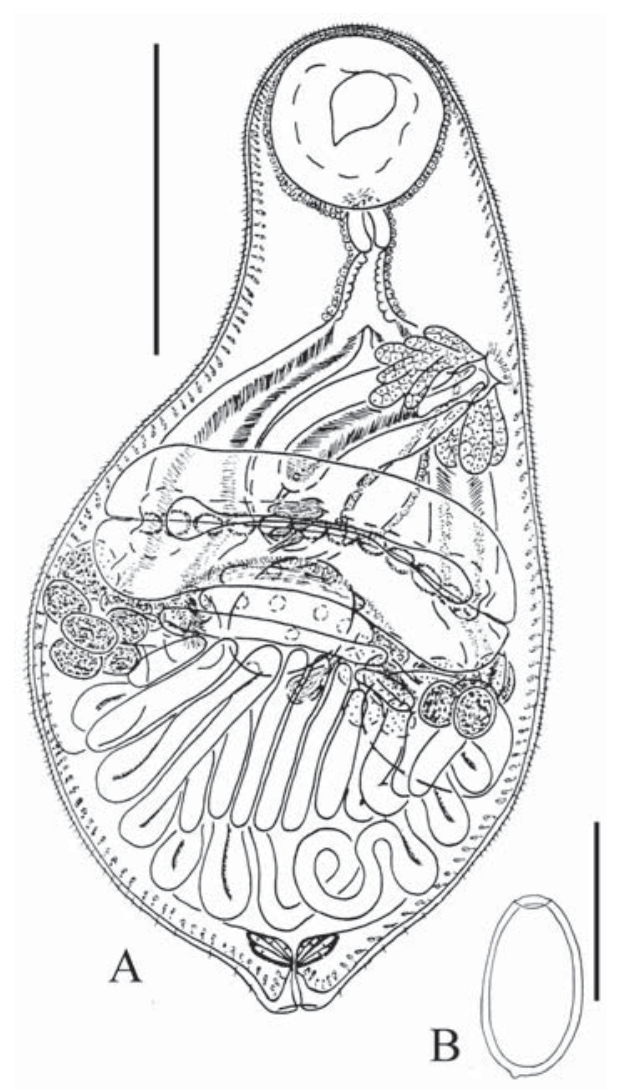

Fig. 5. Proctophantastes sp.

A — general view; B — egg. Scale bars: A $-0.7 \mathrm{~mm}$; B $0.04 \mathrm{~mm}$.

Рис. 5. Proctophantastes sp.

А — общий вид; В — яйцо. Масштаб: А - 0,7 мм; В 0,04 мм.

posterior to level of intestinal bifurcation. Ovary round or suboval, median or submedian, dorsal to posterior margin of ventral sucker. Seminal receptacle sinistral to ovary. Laurer's canal not visible. Hindbody filled with numerous uterine loops. Metraterm surrounded by glandular cells. Eggs operculate. Vitelliarium follicular, in two symmetrical lateral clusters, located just posterior to posterior margin of ventral sucker; left and right clusters containing 8-10 follicles each. Excretory pore terminal; posterior part of excretory bladder surrounded by wide ring of glandular cells.

This parasite is allocated to Proctophantastes Odhner, 1911 because of the presence of two lateral clusters of vitelline follicles, the 
position of testes at the level of vitelliarium, the position of the posterior end of the intestinal caeca at the level of testes and the presence an equatorial dorsal cleft and ventral ridge at the ventral sucker (Bray et al., 2008). This genus includes six valid species: $P$. abyssorum Odhner, 1911, P. brayi Mouahid et al., 2008, P. gillissi (Overstreet et Pritchard, 1977), P. glandulosum (Byrd, 1964), P. infundibulum Kamegai, 1973 and $P$. nettastomatis Machida et al., 2006 (Byrd, 1964; Kamegai, 1973; Overstreet, Pritchard, 1977; Bray, 1987; Bray, Gibson, 1986; Machida et al., 2006; Mouahid et al., 2008). This Proctophantastes sp. differs from all of the other species in the morphology of the ventral equatorial ridge of the ventral sucker. This ridge, in known species is in the form of a continuous muscle torus, but ridge of Proctophantastes $\mathrm{sp}$. is a transverse row of closely adjusted subglobular or oblong muscular protrusions. The taxonomical status of Proctophantastes sp. requires additional material to be provided for further analysis.

\section{Discussion}

Trematodes were found in six of the seven species of fishes examined during the investigation. Only Dissostichus mawsoni and Trematomus hansoni have previously been examined in the Ross Sea (Byrd, 1963, Holloway, Bier, 1968; Holloway, Spence, 1980; Zdzitowiecki et al., 1999). We recorded four species of trematodes from D. mawsoni and T. hansoni, only two of which were previously known from the Ross Sea. Only one species, Postlepidapedon opisthobifurcatum, was recorded from Macrourus whitso$n i$. This parasite was known from this host only in the Weddell Sea (Zdzitowiecki, Cielecka, 1997a; Walter et al., 2002). Published data on parasites of Muraenolepis marmorata exist only for the Indian Ocean sector of the sub-Antarctic waters (Parukhin, Lyadov, 1979; Parukhin, 1989; Gaevskaya, Rodjuk, 1997), although another species of this genus, M. microps, has been examined from the Antarctic waters (the Atlantic sector) (Zdzitowiecki, 1991; Gaevskaya, Rodjuk, 1997; Palm et al., 2007 and others). In the present study,
M. marmorata is described as a new host for the following six species: Lecithaster micropsi, $P$. opisthobifurcatum, Discoverytremagibsoni, Discoverytrema markowskii, Macvicaria muraenolepidis and Proctophantastes sp. With regard to Bathyraja meridionalis and Chionobathyscus dewitti, there are no previous records of these species as host of trematode species.

Twenty-seven determined or undetermined species of trematodes are now known from the present and literature data to occur in fishes from the Ross Sea (Holloway, Bier, 1968; Holloway, Spence, 1980; Zdzitowiecki et al., 1999; Brickle et al., 2005 etc). Otodistomum cestoides, Lecithaster micropsi, Neolepidapedon trematomi, Postlepidapedon opisthobifurcatum, Discoverytrema gibsoni, D. markowskii, Macvicaria muraenolepidis and Helicometra rakusai are now known to be distributed in Antarctic waters more widely than previously believed.

\section{Acknowledgements}

The authors are deeply indebted to S. Kornienko, R. Salamatin, A. Rocka, W. Jeżewski, D. Gibson, R. Bray and T. Cribb for help in searching the literature. The work was partly supported by the RF President's Grant for the support of leading scientific schools \# 2706.2012.4 and RFBR \# 12-04-33045.

\section{References}

Andriyashev A.P. 1986. [Review of bottom fish fauna of the Antarctica] // Trudy Zoologicheskogo Instituta AN SSSR. Vol.153. P.9-45 [in Russian].

Bray R.A. 1987. A revision of the family Zoogonidae Odhner, 1902 (Platyhelminthes: Digenea): subfamily Lepidophyllinae and comments on some aspects of biology // Systematic Parasitology. Vol.9. P.83-123.

Bray R.A., Cribb T.H. 2012. Reorganization of the superfamily Lepocreadioidea Odhner, 1905 based on an inferred molecular phylogeny // Systematic Parasitology. Vol.83. P.169-177.

Bray R.A., Gibson D.I. 1986. The Zoogonidae (Digenea) of fishes from the north-east Atlantic // Bulletin of the British Museum (Natural History) // Zoology. Vol.51. P.127-206.

Bray R.A., Gibson D.I., Jones A. (Eds.). 2008. Keys to the Trematoda, 3. Wallingford UK, The Natural History Museum, London, UK: CABI Publishing. 824 p.

Brickle P., MacKenzie K., Pike A. 2005. Parasites of the Patagonian toothfish, Dissostichus eleginoides Smitt, 
1898, in different parts of the Subantarctic // Polar Biology. Vol.28. P.663-671.

Bush A.O., Lafferty K.D., Lotz J.M., Shostak A.W. 1997. Parasitology meets ecology on its own terms: Margolis et al. revisited // Journal of Parasitology. Vol.83. P.575-583.

Bykhovskaya-Pavlovskaya I.E. 1985. [Parasites of fishes]. Leningrad: Nauka. 121 p. [In Russian].

Byrd M.A. 1963. Helminth parasites of Antarctic vertebrates. Part I. Digenetic trematodes of marine fishes // Proceedings of the Helminthological Society of Washington. Vol.30. P.129-148.

Byrd M.A. 1964. Neosteganoderma glandulosa gen. n., sp. n. (Trematoda: Steganodermatidae) from an Atlantic fish // Proceedings of the Helminthological Society of Washington. Vol.31. P.105-108.

CCAMLR. 2011. The Commission for the Conservation of Antarctic Marine Living Resources Scientific Observer Manual. CCAMLR Document. Australia. Tasmania. Hobart. 141 p.

CCAMLR. 2012. The Commission for the Conservation of Antarctic Marine Living Resources Schedule of Conservation Measures in force 2012/13 season. CCAMLR Document. Australia. Tasmania. Hobart. $300 \mathrm{p}$.

Donnelly J., Torres J.J., Sutton T.T., Simoniello C. 2004. Fishes of the eastern Ross Sea, Antarctica // Polar Biology. Vol.27. P.637-650.

Eastman J.T., Hubold G. 1999. The fish fauna of the Ross Sea, Antarctica // Antarctic Science. Vol.11. P.293304.

Fisher W., Hureau J.-C. (Eds.). 1985. FAO Species identification sheets for fishery purposes. South Ocean: fishing areas 48, 58 and 88 (CCAMLR convention area). Prepared and published with the support of the Commission for the Conservation of Antarctic Marine Living Resources, 2. Roma: FAO. P.235-479.

Gaevskaya A.V., Rodjuk G.N. 1997. [Ecological-geographical characters of parasite fauna of muraenolepids (Pisces: Muraenolepididae)] // Ekologiya moray. Vol.46. P.28-31 [in Russian].

Gibson D.I., Jones A., Bray R.A. (Eds.). 2002. Keys to the Trematoda. 1. Wallingford UK, The Natural History Museum. London. UK. CABI Publishing. 521 p.

Holloway H.L., Bier J.W. 1968. Helicometra antarcticae sp. nov. from Antarctic coastal fishes // Proceedings of the Helminthological Society of Washington. Vol.35. P.30-34.

Holloway H.L., Spence J.A. 1980. Ecology of animal parasites in McMurdo Sound, Antarctica // Comparative Physiology and Ecology. Vol.5. P.262-284.

Jones A., Bray R.A., Gibson D.I. (Eds.). 2005. Keys to the Trematoda, 2. Wallingford UK, The Natural History Museum, London, UK: CABI Publishing. 768 p.

Kamegai S. 1973. Zoogonid trematodes from marine fishes near the Tsushima Islands in the Sea of Japan // The Research Bulletin of the Meguro Parasitological Museum. Vol.7. P.19-23.

Laskowski Z., Rocka A., Zdzitowiecki K., Ghigliotti L., Pisano E. 2005. New data on the occurrence of inter- nal parasitic worms in the Gymnodraco acuticeps and Cygnodraco mawsoni (Bathydraconidae) fish in the Ross Sea, Antarctica // Polish Polar Research. Vol.26. P.37-40.

Leiper R.T., Atkinson E.L. 1914. Helminths of the British Antarctic Expedition, 1910-1913 // Proceedings of the Zoological Society of London. Vol.1. P.222-226.

Leiper R.T., Atkinson E.L. 1915. Parasitic worms with a note on a free-living nematode // British Antarctic ("Terra-Nova") Expedition. Natural History Reports Zoology. Vol.2. P.19-60.

Machida M., Kamegai S., Kuramochi T. 2006. Zoogonidae (Trematoda, Digenea) from fishes of Japanese waters // Bulletin of the National Science Museum. Series A. Vol.32. P.95-104.

Moser M., Cowen R.K. 1991. The effects of periodic eutrophication on parasitism and stock identification of Trematomus bernacchii (Pisces: Nototheniidae) in McMurdo Sound, Antarctica // Journal of Parasitology. Vol.77. P.551-556.

Mouahid G., Faliex E., Allienne J.-F., Cribb T.H. 2008. Proctophantastes brayi, n. sp. (Digenea: Zoogonidae) parasite of the deep-sea fish Polymixia Lowe, 1838 from Vanuatu // Parasitology International. Vol.57. P.25-31.

Orecchia P., Paggi L. 1990. Reperti parassitologici in Chionodraco hamatus e Pagothenia bernacchii delta baia di Terra Nova (Mare di Ross, Antartide) // Parassitologia. Vol.32. P.188.

Overstreet R.M., Pritchard M.H. 1977. Two new zoogonid Digenea from deep-sea fishes in the Gulf of Panama / / Journal of Parasitology. Vol.63. P.840-844.

Palm H.W., Klimpel S., Walter T. 2007. Demersal fish parasite fauna around the South Shetland Islands: high species richness and low host specificity in deep Antarctic waters // Polar Biology. Vol.30. P.15131522.

Parukhin A.M. 1989. [Parasitic worms of bottom fishes of the southern seas]. Kiev: Naukova Dumka. 156 p [in Russian].

Parukhin A.M., Lyadov V.N. 1979. [New genus and species of trematodes, fish parasites in the subantarctic zone of the Indian Ocean] // Zoologicheskiy Zhurnal. Vol.53. P.637-642 [in Russian].

Prudhoe S., Bray R.A. 1973. Digenetic trematodes from fishes // Reports B.A.N.Z. Antarctic Research Expedition, Series B. Vol.8. P.195-225.

Walter T., Palm H.W., Piepiorka S., Rückert S. 2002. Parasites of the Antarctic rattail Macrourus whitsoni Regan, 1913 (Macrouridae, Gadiformes) // Polar Biology. Vol.25. P.633-640.

Zdzitowiecki K. 1987. Digenetic trematodes from the alimentary tract of fishes off South Shetlands (Antarctic) // Acta Parasitologica Polonica. Vol.32. P.219232.

Zdzitowiecki K. 1988. Occurrence of digenetic trematodes in fishes off South Shetlands (Antarctic) // Acta Parasitologica Polonica. Vol.33. P.155-167.

Zdzitowiecki K. 1990. Antarctic representatives of the genus Macvicaria Gibson \& Bray, 1982 (Digenea, 
Opecoelidae), with descriptions of two new species // Systematic Parasitology. Vol.16. P.169-179.

Zdzitowiecki K. 1991. Occurrence of digeneans in open sea fishes off the South Shetland Islands and South Georgia, and a list of fish digeneans in the Antarctic // Polish Polar Research. Vol.12. P.55-72.

Zdzitowiecki K. 1992 Antarctic representatives of the genus Lecithaster Lühe, 1901 (Digenea, Hemiuridae), with the description of a new species // Acta Parasitologica. Vol.37. P.57-63.

Zdzitowiecki K. 1997. Digenea of fishes of the Weddell Sea. IV. Three opecoelid species of the genera Neolebouria, Helicometra and Stenakron // Acta Parasitologica. Vol.42. P.138-143.

Zdzitowiecki K. 1999. Digeneans of the families Opecoelidae and Lepocreadiidae, parasites of Lepidonotothen macrophthalma from the North Scotia Ridge, and remarks on the discrimination of Neolepidapedon magnatestis and N. trematomi // Acta Parasitologica. Vol.44. P.233-240.

Zdzitowiecki K. 2001. New data on the occurrence of fish endoparasitic worms off Adelie Land, Antarctica // Polish Polar Research. Vol.22. P.159-165.
Zdzitowiecki K., Cielecka D. 1997a. Digenea of fishes of the Weddell Sea I. Parasites of Macrourus whitsoni (Gadiformes, Macrouridae) // Acta Parasitologica. Vol.42. P.23-30.

Zdzitowiecki K., Cielecka D. 1997b. Digenea of fishes of the Weddell Sea. III. The Lepocreadiidae (genera Neolepidapedon and Lepidapedon), parasites of Notothenioidea // Acta Parasitologica. Vol.42. P.84-91.

Zdzitowiecki K., Laskowski Z. 2004. Helminths of an Antarctic fish, Notothenia coriiceps, from the Vernadsky Station (Western Antarctica) in comparison with Admiralty Bay (South Shetland Islands) // Helminthologia. Vol.41. P.201-207.

Zdzitowiecki K., Palladino S., Vacchi M. 1999. Preliminary results on Digenea found in fishes in the coastal waters of Terra Nova Bay, Antarctica // Parassitologia. Vol.41. P.575-578.

Zdzitowiecki K., White M.G., Rocka A. 1997. Digenean, monogenean and cestode infection of inshore fish at the South Orkney Islands // Acta Parasitologica. Vol.42. P.18-22.

Responsible editors N.M. Biserova, K.G. Mikhailov 